\title{
Growth and micro-topographical studies of gel grown cholesterol crystals
}

\author{
ANIT ELIZABETH, CYRIAC JOSEPH and M A ITTYACHEN* \\ School of Pure and Applied Physics, Mahatma Gandhi University, Kottayam 686 560, India
}

MS received 23 March 2001; revised 10 May 2001

\begin{abstract}
Cholesterol $\left(\mathrm{C}_{27} \mathrm{H}_{46} \mathrm{O}\right)$ is the most abundant and best-known steroid in the animal kingdom. The in vitro crystallization of this important biomaterial has been attempted by few researchers. Here we are reporting crystallization of pure cholesterol monohydrate crystals in gel medium. It is found that the morphology of the crystals depends on various parameters. The effect of solvent has been studied in detail. The different morphologies observed are fibrous, needle, platelet, dendrite etc. Micro topographical studies have been made and it is found that the crystals grow, at least in the last stage, by spreading of layers. However, at initial stage microcrystals formed and developed into dendrite or needle forms. These one-dimensional crystals developed into platelets and finally thickened. Further studies reveal that micro impurities play a vital role in the development of these crystals as seen by dissolution figures on the crystals. These crystals are characterized by using the XRD and IR spectroscopic methods.
\end{abstract}

Keywords. Cholesterol; gel method; organic solvents; micro-topographical studies; layered growth.

\section{Introduction}

Cholesterol $\left(\mathrm{C}_{27} \mathrm{H}_{46} \mathrm{O}\right)$ is the most abundant and bestknown steroid in animal kingdom. It is found in brain, nerve tissue and cell membranes. It is a major constituent of crystalline material in gallstones (Admirand and Small 1968) and also acts as the precursor of bile acid and steroid hormones. Cholesterol has low solubility in water but it is soluble in organic solvents such as ethanol, methanol and benzene. The solubility of cholesterol in various solvents has been widely studied and reported (Bar et al 1984; Pal and Moulik 1987). Cholesterol was crystallized from different solvents under various conditions and the effect of solvents on the crystal structure was studied (Garti et al 1980, 1981). Gel is an ideal medium to grow biological crystals since its structure is similar to the mucus in the living organisms. The internal surface of the organs in animals is invariably covered with mucus membrane. This material has open structure containing pores of different sizes. These pores can act as nucleation centres for the growth of crystals. Even at low supersaturation, specific molecules can segregate creating critical nuclei to enhance the growth of crystalline materials. Kalkura and Devanarayanan $(1986,1987)$ have grown cholesterol monohydrate crystals in gel medium and showed that these crystals grow in fibrous and platelet forms. In the present work cholesterol is grown in silica gel medium using different solvents. The effect of temperature and concentration on the growth is also studied.

*Author for correspondence

\section{Experimental}

The single test-tube diffusion method (Henisch 1988) was employed for growing cholesterol crystals in the gel medium. The stock solution of specific gravity 1.03 to $1.05 \mathrm{~g} / \mathrm{cc}$ was prepared by dissolving sodium meta-silicate powder in doubly distilled water at room temperature. The solution was filtered and kept in a clean flask. This solution was mixed with acetic acid to adjust the $\mathrm{pH}$ of the solution. By varying the $\mathrm{pH}$ one can control the density of the gel. In the present case gel of $\mathrm{pH} 4-6$ were employed. The resulting solution was mixed with different organic solvents and allowed to gel. The presence of organic solvents in the gel will enhance the growth of crystals in the gel medium. The supernatant solution was prepared by dissolving required amount of cholesterol $(\mathrm{CDH}, \mathrm{AR})$ in corresponding organic solvents.

\section{Results}

\subsection{Effect of different solvents}

In the first case the stock solution was mixed with acetone in $2: 1$ ratio and the resulting solution was allowed to gel in test tubes. A $0.5 \%(\mathrm{w} / \mathrm{v})$ solution of cholesterol in acetone was poured over the set gel and kept for the upper solution to diffuse through the gel medium. After two days of incubation period, very fine crystals appeared in the upper solution. Some of them penetrated through the interface towards the gel medium. The number of narrow 
platelets were increased and invaded complete region of the upper solution within one week. In some cases narrow plate like growth was found to branch out from one strip. These branches were found to be parallel to each other. The nature of growth is shown in figure 1 . The lengths of the grown crystals varied from $1-2.5 \mathrm{~cm}$ and the width was about $2-3 \mathrm{~mm}$. The surface of the platelets shows growth steps one over the other. The magnified photograph of the crystal surface is shown in figure 2 . At the initial stage of the growth the crystals were found to be few in numbers and then increased after 4-5 days. Beside these types of crystals fibrous growth was also observed in the solution and in some cases in the gel medium also.

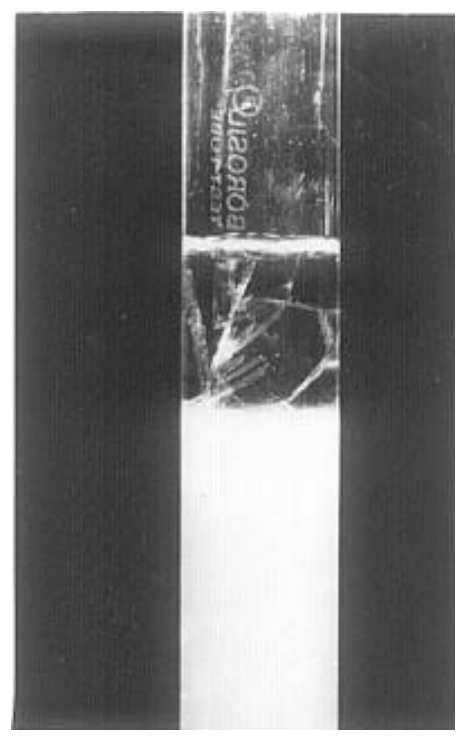

Figure 1. Growth of cholesterol crystal in acetone medium.

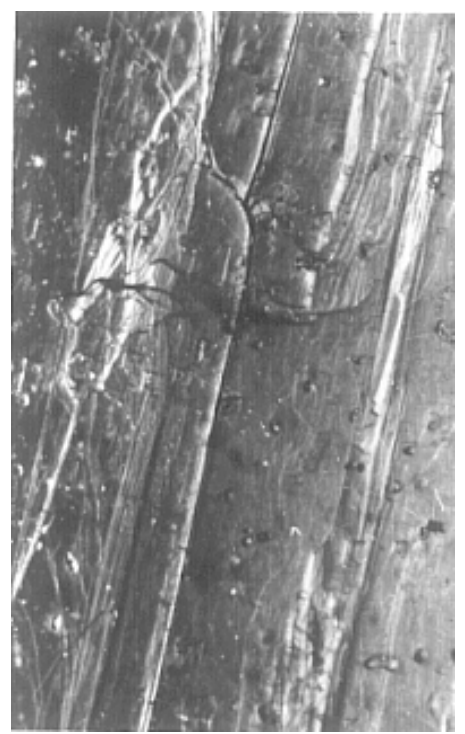

Figure 2. Cholesterol crystal surface with growth steps.
Instead of acetone, ethanol and methanol were used to study the growth of these crystals. When ethanol was used as solvent, thin plate like crystals were found to grow in the supernatant solution and needle like crystals inside the gel. The platelets $($ size $1 \times 1 \mathrm{~cm}$ ) are rectangular in shape. A small region platelet under higher magnification is shown in figure 3 . Some crystals have overgrowth on its surface, the shape of which is same as that of the face. In the gel medium needle like growth was also observed originating from the interface and growing into the gel.

When methanol was used, the nature of growth was different from above. In this case needle like growth was observed in the supernatant solution. The needles were found to originate from the interface and grown vertically

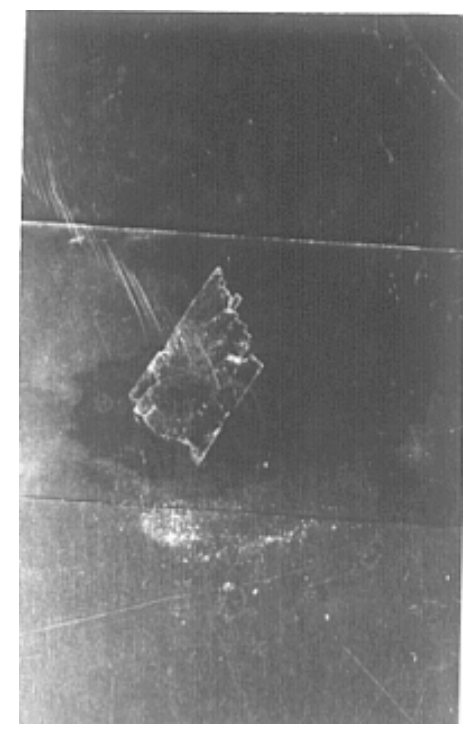

Figure 3. Platelet shaped cholesterol crystal grown in ethanol.

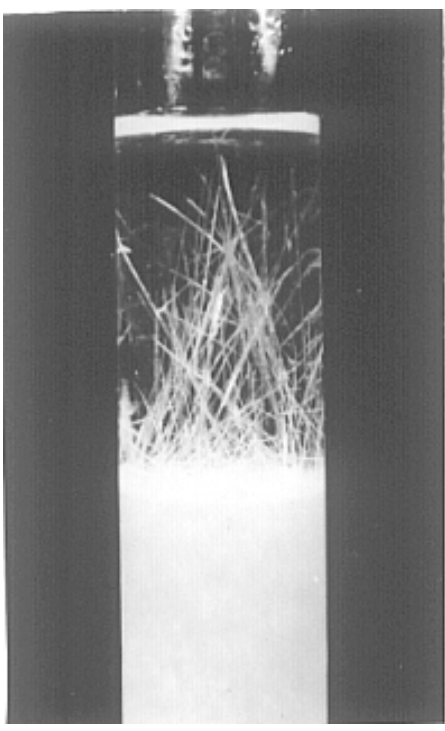

Figure 4. Growth system of cholesterol crystals in methanol. 
upward (figure 4). Few needle like growth was observed in the gel medium also. The growth rate in the solution was very high. The growth started within an hour after the cholesterol solution was poured over the gel. In this case also the needles have invaded complete region of the upper solution. The length of the needle goes up to 4 to $5 \mathrm{~cm}$. This lengthening of the needle depends on the length of the liquid column over the gel. Close observations of these needles reveal that few among these crystals tend to produce branches at an angle of $55^{\circ}$ with the stem. A typical example is shown in figure 5. It is interesting to note that these branches remain dormant in further growth. Needles without branches are also seen. The results observed using different solvents are given in table 1 .

\subsection{Effect of concentration of supernatant solution and growth temperature}

The experiment was repeated by varying the concentration of supernatant solution. Cholesterol dissolved in acetone in different concentrations $(0.25 \%, 0.5 \%, 1 \%)$. When the concentration was high $(1 \%)$, the crystals started growing

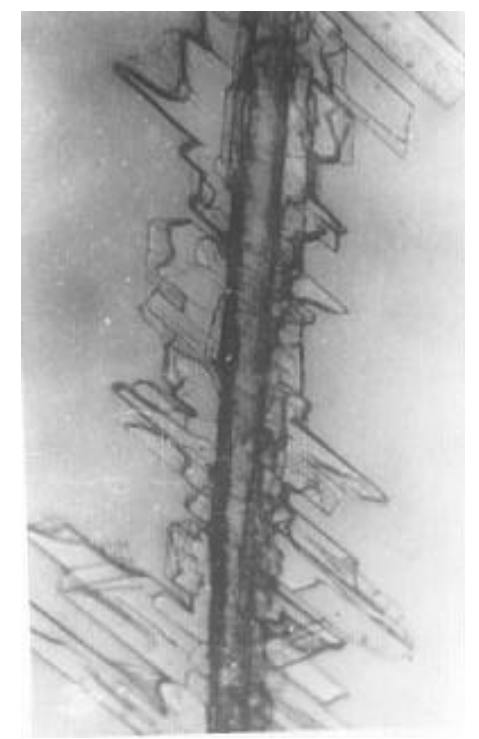

Figure 5. Branched growth on a needle shaped crystal. within $24 \mathrm{~h}$. When the concentration was $0.5 \%$ the growth started within $48 \mathrm{~h}$ and for $0.25 \%$ the growth started within $72 \mathrm{~h}$. The result also shows that the growth density depends on the concentration of the cholesterol solution. As the concentration increases the nucleation density also increases. In the case of methanol and ethanol the same result was observed.

Temperature plays an important role in the crystallization of cholesterol in the human body. The experiments were carried out at different temperatures, at $30^{\circ} \mathrm{C}$ (room temperature) and at $40^{\circ} \mathrm{C}$. The nucleation density was more when the temperature was high. It is reported that the cholesterol deposition occurs in species whose body temperature is higher than $37^{\circ} \mathrm{C}$ (Labowitz 1972). The increased nucleation at high temperature $\left(40^{\circ} \mathrm{C}\right)$ is in agreement with the report.

\section{Discussion}

Growth experiments conducted using different solvents show that the nature of the solvents affects the morphology of the crystals. The crystals grown in methanol are predominantly in the form of needles with branches in crystallographic orientations. In the case of acetone thicker branches are developed while in ethanol platelets of dimensions up to 1-2 cm are obtained.

Detailed microtopographical examinations of the grown crystals reveal that it has thin growth layers parallel to (001) surface. Some crystals are plane without any features. Some are highly mottled. Oriented square pits of flat bottomed and terraced in nature are seen in some crystals (figure 6). Invariably there will be a dark spot at the centre of the pits. Absence of point bottomed pits show that these are not produced by any dislocations. These shallow pits are formed as a result of layer deposition and dissolution.

The observations show that the crystals in the form of platelets grow by two-dimensional nucleation and subsequent spreading of the layers and the layers are parallel to the longer edges of the crystals. Crystallographically oriented pits are due to this layer growth from the edges of the crystals and the subsequent dissolution. The impurity segregation may be aiding the dissolution. Stepped

Table 1. Effect of solvents on the crystallization of cholesterol crystals.

\begin{tabular}{|c|c|c|c|}
\hline Solvent used & Region of crystal formation & Morphology of grown crystals & Size of the crystal \\
\hline \multirow[t]{2}{*}{ Acetone } & Supernatant solution & Lath shaped crystals & $1-3 \mathrm{~cm}$ (length); $2-3 \mathrm{~mm}$ (width) \\
\hline & Inside the gel & Fibrous and needle like growth & $2-3 \mathrm{~mm}$ \\
\hline \multirow[t]{2}{*}{ Ethanol } & Supernatant solution & Plate like growth & $1 \times 1.5 \mathrm{~cm}$ \\
\hline & Inside the gel & Fibrous and dendrite like growth & $2-6 \mathrm{~mm}$ \\
\hline \multirow[t]{2}{*}{ Methanol } & Supernatant solution & Needle like growth & $3-5 \mathrm{~cm}$ \\
\hline & Inside the gel & Needle like growth & $2-6 \mathrm{~mm}$ \\
\hline
\end{tabular}




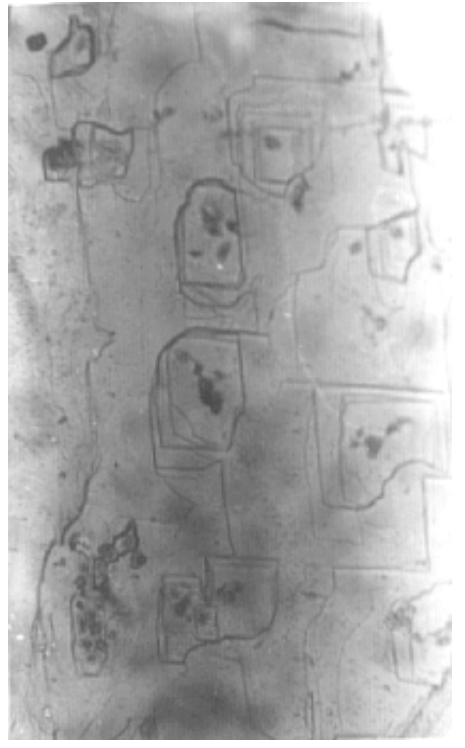

Figure 6. Crystallographically oriented pits on the surface of cholesterol crystal.

pits are again evidence of layer growth. Controlled dissolution experiments have been done by using acetone and found that systematic enlargement of these pits takes place without showing any point bottoms. Critical examinations of featureless surfaces show no evidence of screw dislocation. It can be assumed that cholesterol grow purely by two-dimensional nucleation and layer mechanism.
Characterization has been done by X-ray diffraction and infrared spectroscopy. It is found that the samples grown are crystalline in nature and it is in monohydrate form.

\section{Conclusions}

Crystallization of cholesterol in gel using different organic solvent show that the morphology depends on the type of solvents used. The morphological change is due to the degree of solubility of cholesterol in different solvents. On the basis of micro-topographical observations a model of growth of these crystals is proposed. These crystals grow mainly by two-dimensional nucleation and spreading of layers. It is found that the temperature also influences the rate of growth.

\section{References}

Admirand W H and Small D M 1968 J. Clin. Invest. 471045 Bar L K, Garti N, Sarig S and Bar R 1984 J. Chem. Eng. Data 29440

Garti N, Karpuj L and Sarig S 1980 Thermochim. Acta 35343

Garti N, Karpuj L and Sarig S 1981 J. Lipid Res. 22785

Henisch H K 1988 Crystal growth in gels and Liesegang rings (Cambridge: Cambridge University Press)

Kalkura S N and Devanarayanan S 1986 J. Mater. Sci. Lett. 5741

Kalkura S N and Devanarayanan S 1987 J. Cryst. Growth 83446

Labowitz L C 1972 Thermochim. Acta 3419

Pal S and Moulik S P 1987 Indian J. Biochem. Biophys. 2424 